\title{
Correction: Assay to rapidly screen for immunoglobulin light chain glycosylation: a potential path to earlier AL diagnosis for a subset of patients
}

\author{
Sanjay Kumar ${ }^{1} \cdot$ David Murray $^{2} \cdot$ Surendra Dasari $^{3} \cdot$ Paolo Milani $^{4} \cdot$ David Barnidge $^{2} \cdot$ Benjamin Madden $^{5}$. \\ Taxiarchis Kourelis ${ }^{1} \cdot$ Bonnie Arendt ${ }^{2} \cdot$ Giampaolo Merlini $^{4} \cdot$ Marina Ramirez-Alvarado $^{6}$ - Angela Dispenzieri ${ }^{1,2}$
}

Published online: 28 February 2019

c) Springer Nature Limited 2019

Correction to: Leukemia (2019)33: 254-257; https://doi.org/10.1038/s41375-018-0194-x; published online 6 July 2018

Following the publication of this article, the authors noted that Patrick M. Vanderboom was inadvertently omitted from the author list. The correct author list is as follows: Sanjay Kumar, David Murray, Surendra Dasari, Paolo Milani, David Barnidge, Benjamin Madden, Patrick M. Vanderboom, Taxiarchis Kourelis, Bonnie Arendt, Giampaolo Merlini, Marina Ramirez-Alvarado, Angela Dispenzieri. Patrick M. Vanderboom is affiliated with the Mayo Clinic in Rochester, MN.
Angela Dispenzieri

Dispenzieri.Angela@mayo.edu

Division of Hematology, Mayo Clinic, Rochester, MN, USA

2 Department of Laboratory Medicine, Mayo Clinic, Rochester, MN, USA

3 Department of Health Science, Mayo Clinic, Rochester, MN, USA

4 Amyloidosis Research and Treatment Center, Fondazione IRCCS Policlinico San Matteo and Department of Molecular Medicine, University of Pavia, Pavia, Italy

5 Medical Genomics Facility, Proteomics Core, Mayo Clinic, Rochester, MN, USA

6 Departments of Biochemistry and Molecular Biology, Mayo Clinic, Rochester, MN, USA 